Historic, Archive Document

Do not assume content reflects current scientific knowledge, policies, or practices. 



\section{Special Contract Prices of High Class Flower Seeds \\ 1}

QUALITY - is my motto. Twenty years' experience in the horticultural and agricultural business has taught me the full meaning of the word.

PRICES-1 trust the unusual low prices quoted in this list will not cause you to experience the common feeling of distrust for a cheap priced article.

I ASSURE YOU-my price represents what 1 consider is the proper value of a given crop not influenced by other merchants' valuations.

TERMS-f. o. b. New York, 2 per cent. on receipt of goods. 60 days Net. I exercise the greatest care in growing and harvesting my seeds, roguing my crops under personal supervision. It must be understood, however, that I warrant neither description, growth, nor productiveness of any seeds I send out, nor will I hold myself responsible for the crops raised therefrom. If not accepted on these terms they must be returned at once.

\section{FOR DELIVERY AFTER HARVEST 1915}

All contract orders booked subject to usual pro-rata crop delivery. That land sufficient for your wants may be reserved I ask you to place orders at earliest

\section{ASTERS}

The quality of my Asters is unsurpassed; they will satisfy the most critical trade.

\section{GIANT HOHENZOLLERN}

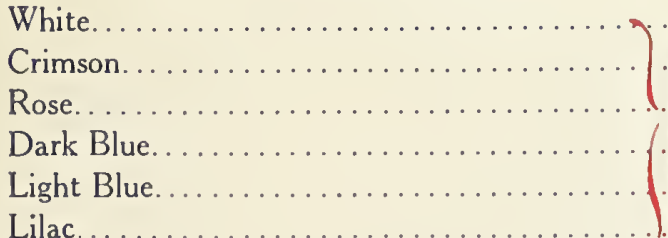

Filac.........

\section{AMERICAN BRANCHING}

Without doubt the finest florist variety; long stem; large, double flower; late.

White.

Flesh Pink, or Shell Pink

Rose-Deep Pink.

Crimson.

Purple-Dark Blue.

Lavender-Light Blue.

Blush-Pale Pink

Special Mixed.

\section{TRUFFAUT'S PERFECTION}

White.

\section{(Paeony-flowered)}

Crimson

Dark Blue

Light Blue.
Lb. $\mathrm{Oz}$

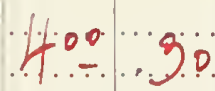

$350 \cdots 30$

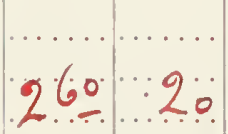

\section{VICTORIA}

White

Rose.

Dark Blue.

Light Blue.

Crimson. .

Finest Mixed.

\section{QUEEN OF THE MARKET}

White....

Light Blue.

Dark Blue.

Crimson. .

Rose.

Scarlet.

Finest Mixed

GIANT COMET

(Selected)

White

Crimson

Rose.

Dark Blue

Light Blue.

Mixed.
Lb. $\mathrm{Oz}$

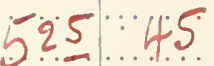

$5: 0 \cdots 40$

$550 \cdots 45$

$6.00 \therefore 50$

$5.00 \therefore 40$

$45 . \ldots 35$

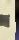




\section{ASTERS-Continued}

MISCELLANEOUS VARIETIES

Mikado Pink.

Mikado White

Purity.

Lavender Gem

Sunset.

Violet King

Rose King.

White King

\section{ANTIRRHINUM}

GIANT FLOWERING

Queen Victoria. Large, pure white.

Firefly. Crimson and yellow.

Luteum. Pure yellow.

Galathee. Reddish purple and white

Brilliant. Blood red.

Giant Flowering. Finest mixed.

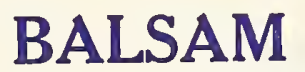

HOY'S IMPROVED

DOUBLE CAMELLIA FLOWERED

White

Rose.

Light Pink.

Purple.

Lilac.

Scarlet

Crimson

Finest Mixed

\section{CALENDULA}

HOY'S IMPROVED

SCOTCH MARIGOLD

Sulphur Queen...

Prince of Orange.

\section{COSMOS}

\section{LATE MAMMOTH FLOWERING}

White.

Pink.

Crimson.

Lady Lenox. Pink.

Lady Lenox. White.

Mammoth Flowering Mixed.
Lb. $\quad \mathrm{Oz}$

$5.00 \cdot 40$

8.00 .215

$10.00 .1: 00$

8.00775

1.06

1.00

$300 \quad 25$

KLONDYKE

New Orange Yellow.

3.750

\section{DIMORPHOTHECA}

Aurantiaca.

Aurantiaca Hybrids.

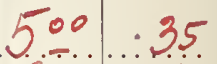

$650 \ldots 45$

\section{ESCHSCHOLTZIA}

(California Poppy)

(One of my great specialties)

California Alba. White.

California. Yellow.

Golden West. Fine yellow.

Rose Cardinal.

Mandarin.

Burbank's Crimson

Carmine King.

Crocea. Double yellow

Crocea. Yellow.

Hoy's Special Mixed.

\section{GODETIA}

Gloriosa (Lord Roberts).

Crimson Glow.

Duchess of Albany

$1.50 \quad 10$

Grandiflora Rosea.

$200 \div 15$

Lady Albemarle.

1. 50 ....10

The Bride.

$1.00 \ldots 10$

$250: 20$

Choice Mixed 
C. C. HOY - Box 93 Station L - BROOKLYN, NEW YORK, U. S. A.

\section{MIGNONETTE}

My strains of Mignonette are attended to with the greatest care.

Allen's Defiance.

Bismarck.

Golden Machet.

Golden Queen

Machet Extra.

Victoria Red.

Miles Spiral.

Parsons' White.

Ruby.

Hoy's Choice Mixed

\section{MARIGOLD}

I save seed only from largest and finest shaped flowers.

\section{HOY'S GIANT FLOWERING AFRICAN}

Lemon Queen...................... 50

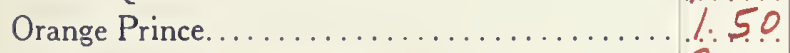

Eldorado. Orange quilled............

Special Mixed.

Legion of Honor.

\section{FRENCH}

Dwarf Mixed.

\section{PANSY}

When comparing the prices on my named Pansies, please consider I offer only the Giant Flowering types.

\section{GIANT TRIMARDEAU}

Lord Beaconsfield.

Fire King.

Emperor William.

Adonis

Yellow Gem. Pure Yellow.

Snowlake. Pure White

White, Dark Eye.

Yellow, Dark Eye

Hoy's Giant Trimardeau Mixed.

Large Flowering French and English Mixed....................

\section{POPPY}

Hoy's Improved Shirley Mixed.

American Flag.

The Bride.

Admiral.

Mikado.

White Swan.

Mephisto.

Miss Sherwood

English Scarlet.

Glaucum

Carnation Flowered Mixed

Paeony Flowered Mixed.

Ranunculus Flowered Mixed

\section{VERBENA}

GIANT FLOWERING

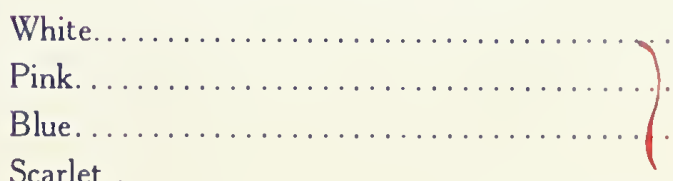

Scarlet

Yellow

Striped

Auricula Eye (Special)

Giant Flowering Mixed.

\section{ZINNIA}

\section{GRANDIFLORA ROBUSTA PLENISSIMA} (Improved Giant Flowering)

White.

Scarlet

Crimson.

Golden Yellow

Purple.

Flesh Pink

Special Mixed.
Lb. $\mathrm{Oz}$.

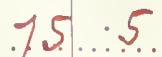

$1.5: 5$

$150 \ldots .5$

Ho

- 40.3

$301 \cdot 5$

$50 \cdots 5$

\% 50.18

$2.00 \cdot 15$

40.5

40.5

$60: 5$
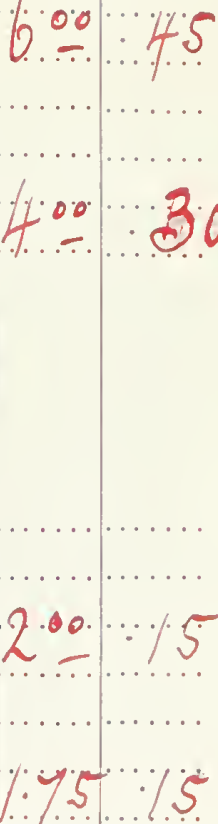

\section{JAPANESE FLOWER SEEDS}

HOY'S JAPANESE GROWN

Convolvulus Imperial Japanese Selected Mixed ...

Lb. Oz.

Convolvulus Imperial Japanese New Giant

Flowering Mixed.

Clematis Paniculata. $\therefore 80 \cdots 10$

$2.50 \cdot 20$

$3.50 \ldots 25$

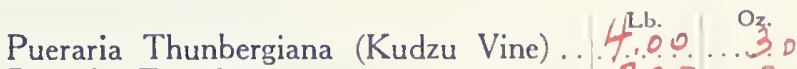
Physalis Franchetti.............. 3.25 ...2. Rosa Polyanthus Multiflora ............. 2.5.0... 2.0 Scabious Japonica .................... 3.5. 5.25 Dianthus Heddewegii f. pl. (Double 2.50 20 


\begin{tabular}{|c|c|c|c|c|}
\hline & 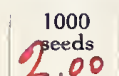 & $\begin{array}{l}100 \\
\text { segds } \\
.2\end{array}$ & Celosia Cristata, Glasgow Prize. & 5.00 \\
\hline $\begin{array}{l}\text { Asparagus Plumosus Nanus. } \\
\text { Phoenix Reclinata......... }\end{array}$ & $\begin{array}{l}2.00 \\
3.00\end{array}$ & $\therefore 25$ & Cobaea Scandens.............. & 3.25 \\
\hline $\begin{array}{l}\text { Phoenix Reclinata... } \\
\text { Phoenix Canariensis. }\end{array}$ & 1.25 & & Coreopsis Lanceolata......... & $2 \cdot 00$ \\
\hline Erythea Armata. Blue Gum & $7 \therefore 00$ & $\therefore 80$ & Delphinium Cardinale. & - \\
\hline paragus Sprengeri.. & 2.25 & 20 & $\begin{array}{l}\text { Dracaena Indivisa. . } \\
\text { Dracaena Australis. }\end{array}$ & $\begin{array}{l}1.50 \\
2.50\end{array}$ \\
\hline Alyssum Maritimum (Sweet) & 1.00 & $\therefore 10$. & Grevillea Robusta... & 4.50 \\
\hline Alyssum Little Gem... & 1.25 & $\therefore 10$ & Smilax ........... & 2.25 \\
\hline
\end{tabular}

\section{Hoy's Flower Seeds in Mixtures}

The following is a list of mixtures which are grown as mixtures but receive good culture and thorough roguing of all objectionable colors. Special quotations for large quantities

Tall, Finest Mixed

ANTIRRHINUM

ASTER

Early Flowering Mixed

BALSAM

Finest Double Mixed.

\section{CANDYTUFT}

Choiee Mixed.

\section{CALENDULA}

Fine Mixed. .

Marguerite Mixed

\section{CARNATION}

CENTAUREA

Cyanus Single Mixed.

Cyanus Double Mixed.

COCKSCOMB

Dwarf Finest Mixed. .

\section{CONVOLVULUS}

Tall Mixed.

\section{COSMOS}

Choice Mixed.

DIANTHUS (Pinks)

Chinese Double Mixed.

\section{ESCHSCHOLTZIA}

Mixed Varieties.

(California Poppy)

\section{GODETIA}

Speeial Mixed

\section{HELICHR YSUM}

(Everlasting Flowers)

Monstrosum Double Mixed.

\section{LARKSPUR}

Tall Rocket Mixed...

Dwarf Rocket Mixed...

\section{MARIGOLD}

Tall African Double Mixed.

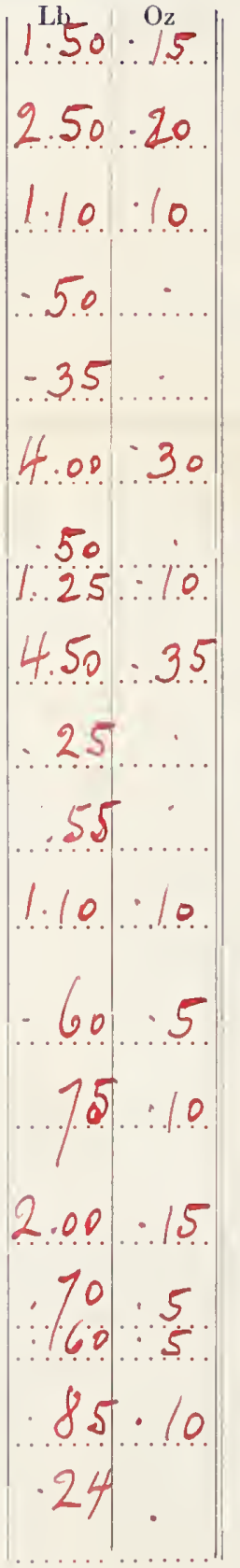

Grandiflora Odorata.

MIGNONETTE

\section{NASTURTIUM}

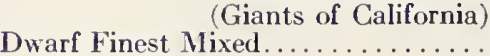

Lb.

28

Tall Finest Mixed.

NIGELLA

Best Mixed.

\section{PANSY}

Hoy's Speeial Mixture.

PETUNIA

Hybrida Finest Mixed.

POPPY

Best Shirley Mixed.

\section{PHLOX}

Drummondi Tall Mixed.......

Drummondi Dwarf Mixed.

23

Single Mixed........

PORTULACA

SCABIOUS

Tall Double Mixed..

Dwarf Double Mixed.

\section{SALPIGLOSSIS}

Variabilis Grandiflora Mixed.

Emperor Giant Mixed.

SALVIA

Splendens....

STOCKS

Dwarf Ten Weeks Mixed.

\section{SWEET WILLIAM}

Single Finest Mixed.

Double Finest Mixed.

SWEET PEAS

Eekford's Large Flowering Mixed.

Itybrida Choiee Mixed.

$$
\text { VERBENA }
$$

WALLFLOWER

Single Mixed.

ZINNIA

$8.75 \ldots .85$

450.240

45

2 5. 20

$\therefore 35$

Tall Double Mixed...........................25 .. 15

Dwarf Double Mixed...................... 